\title{
PIERIS E BEGLIANO: VILLAGGI MEDIEVALI DEL BASSO ISONZO DALL'INCERTA IDENTITÀ
}

Sulla riva sinistra dell'Isonzo e non lontano dalla sua foce si situano alcuni paesi caratterizzati nel basso medioevo da un popolamento misto, sloveno e romanzo, con una certa prevalenza del primo elemento etnico. I paesi sono Pieris, Begliano e San Canziano, con l'appendice di Isola; quest'ultima zona fino al sec. XVI era solo una grande selva, di proprietà della lontana abbazia di Moggio, non abitata ma ben frequentata da boscaioli, cacciatori e pescatori.

La situazione linguistica che abbiamo evocato non era limitata ai tre paesi ma si allargava a tutto il Territorio di Monfalcone e si modificò solo fra la seconda metà del sec. XVI e la prima del successivo, come conseguenza di vasti e complessi eventi storici. ${ }^{1}$ In un periodo incerto ma fra i secoli XV e XVI emerse l'etnico bisiàc che caratterizzerà in epoca moderna il Monfalconese e fin dall'inizio venne identificato nell'area romanza come una varietà «slava». A questo proposito è del massimo interesse per la storia linguistica del Friuli orientale una testimonianza emersa ultimamente: 1462-1464 Sclabonus sive bisiacus. ${ }^{2}$ Va detto che non si trattava probabilmente del vecchio strato etnico sloveno di quel territorio conosciuto nel medioevo come Ultraisoncium ('Oltre il fiume Isonzo'), bensì di immigrati dai paesi balcanici che si mescolarono qui ad altri, provenienti dalle regioni padane.

Il problema della datazione degli originari stanziamenti slavo-alpini o sloveni in Friuli non è stato risolto. Una tradizione storiografica li datò fra i secoli X e XI, come conseguenza delle scorrerie ungariche e del relativo ripopolamento voluto dalle autorità patriarcali. Alcuni toponimi però farebbero pensare a due ondate, una altomedievale (slavo-alpina) composta da pastori ed una frammista alla parte romanza all'epoca dei grandi dissodamenti (ronchi) e della fondazione di ville nove fra la fine del sec. $\mathrm{X}$ ed il sec. XII. Alcuni nomi di luogo infatti, come Bertiolo (ant. Bratiul), Biauzzo (ant. Blaguz), Luvic, Luvidrago, Pradigoi, Visco, Turriaco (ed altri: Puntin 2009) potrebbero risalire all'alto medioevo. Il Monfalconese è un angolo di Friuli stretto

\footnotetext{
* Via Indipendenza/Cadurlina 21, 33050, Fiumicello (Ud); arctomp@gmail.com

1 A cominciare dalle incursioni turche, da una crisi demografica e da una forte immigrazione da Veneto, Lombardia, Istria e Dalmazia; da questi grossi movimenti emerse, come succede spesso, la necessità di una lingua franca, che nell'Alto Adriatico e in territorio veneziano non poteva non essere un dialetto a base veneta. Dialetto che in seguito venne denominato con un termine slavo, bisiàc, che attende ancora una convincente etimologia (P 2005 e 2010).

2 Luogotenente alla Patria del Friuli, b. 32, Processi 1462/1464, c. 100r (ASVe).
} 
fra l'Isonzo ed il Carso, quest'ultimo popolato da sloveni già in età altomedievale; per cui è plausibile che forti gruppi sloveni abbiano colonizzato alcune zone fino alle rive isontine già fra i secoli VII e VIII. Selz, Cassegliano, Staranzano, Turriaco, Sagrado $^{3}$ sono certamente fra queste. In pratica il territorio di Monfalcone andrebbe compreso in quelli della vasta Slavia submersa. In particolare il caso delle tre località prossime, Pieris, Begliano e San Canziano è complesso e interessante da studiare. Pieris ${ }^{4}$ è un paese situato su quelle che sono le rive dell'Isonzo almeno dal sec. XIII ${ }^{5}$ e rappresentava una comunità autonoma che una strada divideva nel campo religioso fra due antiche pievi, S. Pietro (per la parte ad ovest) e S. Canziano (per la parte ad est). Begliano fu un villaggio da sempre sottoposto in spirituali ed in temporali alla vicina pieve di S. Canziano. ${ }^{6}$

La ricerca di documenti che riportassero personali e soprannomi medievali del Monfalconese, iniziata alla fine degli anni novanta, ha dato finora pochi frutti e questo probabilmente perché a differenza di altre zone dello stato patriarcale (l'Udinese, il Cividalese, la zona collinare ecc.) questo angolo di sud-est era importante solo per i tre passi sull'Isonzo, per la Muda del confine ${ }^{7}$ della Patria del Friuli e per la rocca su un colle sovrastante la terra murata di Monfalcone.

Per i toponimi siamo stati più fortunati, considerato che per le questioni di proprietà e di identificazione di terreni e boschi, molti nomi medievali si sono conservati negli atti notarili dei secoli successivi e nei catasti moderni.

A Pieris la divisione del paese a cui abbiamo fatto cenno non influiva sull'onomastica e da una parte e dall'altra troviamo sia personali sloveni (secc. XV-XVI: Blagogna, Blasichio, Bratuç, Gorsé, Greghé, Jursé, Juvaniz, Polcher, Semiç/Simez, Vidiz, Zachoniano Bisiach) che friulani (Camozium, Claudana, Domenie, Georginis, Johannes Ladin, Cussa, Pontel, Lombrena, Pauli).

3 Il primo è chiaramente un diminutivo di selo, il secondo deriverà da uno slavo *Kosovljane (di etimo sub iudice), il terzo (nel medioevo sempre Stranzano) viene probabilmente da un Stran(i)čane 'coloro che abitano dall'altra parte' (di un limite giuridico o fisico o di entrambi). Il quarto è uno dei tanti che alludono allo zoonimo slov. tur 'uro, bisonte' come Turjak (Slo), Turjak (BiH) ecc. Il quinto fa parte della serie che continua lo sloveno *Za-grad-.

4 XII sec. Actum apud Villam, quae dicitur Petra, in prato quodam; XIII sec. in Petris; de Petris; Pieres; XIV sec. villa de Petrie que in theutonico Steindorf nuncupatur; in Pedris; in Piedris et in Ariis; Bonnes de Petris... in Pyeris; Piedris 25 foci; XV sec. Pieris oltra Lusinç; Pieris focos 10 (P 2010).

5 L'alveo del fiume ha subito diversi cambiamenti nel corso del medioevo e da una attestazione trecentesca che parla di Lisoncius vetus sembra di capire che scorresse più ad ovest nel mezzo del vicino paese di Fiumicello. Tutti i fiumi dell'alto Adriatico si andrebbero spostando progressivamente verso est, se non fossero stati fermati da canalizzazioni e bonifiche secolari.

6819 vico sanctorum Cantianorum; XIII sec. de garritu... de ronchis, villa S. Cassiani seu Canciani; XIV sec. in villa S. Canciani; Sand Cancian bey Lisoncz; XV sec. certos redditos in Sancto Canciano et Foglano; in villa Sancti Canciani ultra Osontium, 1466-67 S. Cancianus... focos 12 (P 2010).

7 La Muda è un termine storico che indicava i posti «dove si pagava un dazio doganale o locale sulle merci» (NP: 625). 
Ma si deve ribadire per il Friuli il concetto così ben espresso dal Kronsteiner nel 1981 per l'Austria: chi portava un nome slavo era sicuramente uno sloveno ma non si può dire altrettanto di chi portava nomi generici cristiani o nomi romanzi. ${ }^{8}$ Abbiamo molti esempi di sloveni che nel medioevo portavano nomi romanzi o germanici («di moda» per lunghi secoli nell'ambiente friulano). Gli stessi argomenti valgono in pratica anche per i toponimi, sparsi confusamente nel territorio del villaggio. Sono sloveni come Agrada, Blasizi, Calici, Caminizze, Doja, Dribosa, Juvaniz, Mlacha, Orechovez, Pojane, Raje, Ruppa, Sabornizza, Studensi, Uerbize. Sono friulani Pièris, Chiaròdis, Clapàzi, Dreàt, Rònchija, Vinchiaràda (P 2010): ma come si può osservare anche oggi nella Slavia Friulana i toponimi friulani possono derivare da prestiti lessicali o da immigrati di lingua romanza. ${ }^{9}$

A colpo d'occhio si nota a Pieris una certa prevalenza di nomi di persone edi luoghi di derivazione slovena, a parte il fatto che il toponimo principale è chiaramente friulano, Pièris ('pietre'). Anche in documenti pubblicati negli ultimi anni, quando si tratta di questo paese emerge sempre questo lato «composito». Nell'anno 1321 vengono attestati due contadini di Pieris (Gianni: 378) che lavorano la terra di un nobile abitante in un'altra zona del Friuli: Thomasium e Byeliç. Del primo, un nome cristiano, non si può dir nulla sulla sua identità linguistica: poteva essere di lingua romanza o slava. Del secondo invece siamo certi che fosse sloveno e il particolare vocalismo del nome fa pensare alle tendenze di molti dialetti occidentali, ancora oggi ben presenti.

$\mathrm{Su}$ una strada che dal passo sull'Isonzo di Pieris portava verso Monfalcone e a poca distanza troviamo il paese di Begliano, il cui nome possiede attestazioni a cominciare dal sec. XII. ${ }^{10}$ A Begliano le poche testimonianze medievali, sia onomastiche che toponimiche, vedono una netta prevalenza dell'elemento sloveno: XIV sec. Antonius q. Drusiruai, ${ }^{11}$ Panger, Stoyna de Begliano, Crisman; XV-XVI sec. Johannes Besch, Janze filius Marini, Marini de Beyacho, Petrich, Popuz, Schocho, Juanj Strazay, Martin Straçai, Viman. Risultavano così friulani solo i nomi Baraçuti, de Clement e Mathio Sclopeterio. Toponimi: Carlisa, Crugliza, Dolina, Dusiza, Grubia, Grudenz, Gumiliza, Gusa, Marchviza, Melacche, Nograda, Patoch, Pojana, Prosecha, Prosecha

8 Wir dürfen mit guten Argumenten behaupten, dass der Träger eines slawischen Namens mit unbedeutenden Ausnahmen auch Slawe war... Dies gilt nicht für die deutschen Personennamen aus diesen Kontaktzonen. Es kommt kaum vor, dass ein Deutscher [o un latino] einen slawischen Namen trug, wohl aber dass Slawen deutsche [o nomi latini cristiani e nomi romanzi] Namen trugen (Kronsteiner 1981: 176).

9 Cfr. a Resia i toponimi Bork, Fasál, Kontovél, Máana, Part, Pišánda, Pláča, Runkát, Sadín, Šcafaròt, Sjéja, Štrijúnawo (Dapit 1998).

101138 de Beliani; 1291 in Beliano ultra Isontium; 1321 Beglano, 1332 de Beglano; 1341 Beliano; 1356 Begliano; 1366 in Belano; 1372 Belano; 1447-1448 de Beyacho; 1460 de Beglyano; 1483 in Beglan oltra Lusins; 1490 in Beglan oltra Isonzo, in Belgian oltra Lusonz; 1494 in Villa Beglani ultra Jsontium; 1495-1501 villa Begliana (P 2010).

11 Probabilmente si tratta di un nome sloveno mal scritto nel testo originale: forse *Drusivuoi per un *Družigoi. 
scavezzada, Riboliza, Rupa, Vogri. Anche qui pochi friulanismi, Lòbia, Paniài e Strada dei Ciaròdis. ${ }^{12}$

Il toponimo Begliano a differenza di Pieris resta un caso interessante e controverso, almeno a giudicare dalla posizione di certi studiosi che continuano a ritenerlo un classico prediale romano, da Bellius. Formalmente questa posizione è ineccepibile, dato che il gentilizio o cognomen è attestato in iscrizioni latine (anche Abellius $^{13}$ ). Ma chi scrive dopo un periodo di indecisione ora propende decisamente per la soluzione slavistica. Begliano fa il paio in regione con Beano nel circondario di Codroipo, dove ci fu la più forte colonizzazione slavo-alpina o slovena, forse entrambe in due ondate diverse. ${ }^{14}$ In ambedue le aree dunque i due toponimi, per i quali si registra la stessa dizione friulana Beàn, erano «immersi» in ambienti slavizzati. $\mathrm{Va}$ anche considerato che la coppia Begliano/Beano rappresenta praticamente un unicum in Italia, dove le forme prevalenti si basano piuttosto su Bellus (cfr. Bellano e Bellagio in Lombardia).

È più plausibile l'ipotesi della derivazione da un nome slavo precristiano Beljan/ Beyian/Beyam (con la radice panslava bel- 'bianco'), attestato già nel XIV secolo a Trieste (Merkù 1996: 453) e presente ancora nell'onomastica slovena (sec. XVI Stephano Begliano a Nimis, Bejan a Faedis ${ }^{15}$ ) e friulana del Friuli orientale: il cognome

12 Tutti i personali e toponimi presentati qui sopra provengono da un lavoro dell'autore (P 2010), dove viene proposta l'etimologia più plausibile. Qui sono stati ripresi solo per mostrare al lettore di primo acchito la situazione etno-linguistica probabile della zona. In parte sono stati trascritti da documenti posteriori al medioevo ma nel Monfalconese è implicito che dopo il sec. XVI nessuno poteva dare questi nomi a prati e campi, considerata la profonda venetizzazione del distretto. Anche fra i cognomi dopo tale periodo vi fu un forte ricambio.

13 Ma un Abellius avrebbe avuto un esito diverso nei dialetti romanzi della regione: *Daveàn, *Naveàn. In Gallia è attestato pure il nomen Bellianus (Pierre-Henri 1923: 25), ma in questo caso bisognerebbe pensare che un prediale di quelli cosiddetti a grado zero (senza formante), rarissimi, si sia fissato due volte a non molta distanza l'uno dall'altro.

14 Non serve qui insistere sulla toponomastica slava del Codroipese, già ampiamente studiata (Biauzzo, Glaunicco, Goricizza, Gorizzo, Gradisca, Gradiscutta, Jutizzo, Lonca, Moraulis, Passariano, Passarizza, Zompicchia). Annotiamo solo una novità, ancora poco conosciuta in Friuli. Secondo Silvo Torkar i toponimi sloveni della serie Preserje deriverebbero da un *Prě-syr-jane, (prě come slov. mod. pred 'davanti a', syr 'umidità, bassura erbosa molle'; Torkar 2007: 481484). Quattro toponimi friulani, di cui due nei pressi di Codroipo, farebbero parte della serie: Passariano (1176 Preseriano), Persereano (1166 Presareian), Perserian di Campolongo e Passarizza (*Po-syrica?).

15 Sv. Begliano (SAC 2). Bejan è uno degli antichi nomi di casato dialettali della famiglia Tracogna, in uso a Canebola/Čeniebola, villaggio slovenofono posto nella parte montana del Comune di Faedis (Ud). A Verzegnis e a Venzone fra i secoli XVI e XVII vengono attestati i cognomi Bilianj e Biliano, forse immigrati dalla zona prealpina orientale o dal Canal del Ferro. Ma questi due ultimi cognomi hanno probabilmente origine diversa da quelli della serie Begliano. 
Bean del Monfalconese, ${ }^{16}$ il nome di casato Beàn a Gemona. ${ }^{17}$ Significativi risultano pure gli etnici dialettali sloveni di Resiutta, Bijäni, e di Uccea (Resia), Biejani (così nel dialetto sloveno tersko delle Valli del Torre). ${ }^{18}$

Fuori dalla regione troviamo i cognomi croati Bilan, Bjelanovic, ${ }^{19}$ Belanov in altre regioni slavofone e Bejan nei distretti romeni di Iaşi e Galați. ${ }^{20} \mathrm{Si}$ vedano ancora, nel campo toponomastico, il nome dialettale sloveno della località Vellach in Carinzia, Beljani (Kranzmayer 1958: 67), un importante oronimo della Slovacchia, i Beljanske Tatry, i nomi delle città di Bielany in Polonia e di Bijeljani in Erzegovina. Se per quanto riguarda la semantica, il toponimo Begliano/Beàn dovesse celare non tanto descrizioni d'ambiente ('acque bianche' ecc.) bensì un antico personale e si ipotizzasse una sua datazione precedente all'anno $800,{ }^{21}$ va ricordato che il Bezlaj ebbe a dare alcune indicazioni, per cui al primo posto verrebbero le denominazioni di insediamenti «da nomi personali». Un secondo gruppo sarebbe rappresentato dai dendronimi (es. fiume Sdobba) non alterati con ulteriori composizioni; un terzo gruppo importante sarebbe formato dai toponimi recepiti dagli Slavi dal precedente strato latino e prelatino (Merkù 2001: 706-711).

Quando si argomenta riguardo alla derivazione slava del nome della località Begliano va infine tenuto conto del fatto non certo secondario che l'antica pieve di S. Canziano (con annesso monasteriolo ${ }^{22}$ ), da cui Begliano dipendeva, mostra anch'essa una onomastica ed una toponimia bassomedievali in gran parte slovene. ${ }^{23}$

16 Il cognome Bean del basso Isontino sembra autoctono e indicare in origine «uno originario di Begliano/Beàn». Diverso è il caso di un cognome Bean del Rovigotto: potrebbe derivare da un *Belan (che bèla come una pecora, da cui dialettale veneto beàn 'sciocco'), con lenizione della liquida. Il cognome Bellan del Rovigotto invece dove la liquida permane nella dizione popolare (Belàn) potrebbe rappresentare un etnico da qualche località con un nome composto, avendo come secondo elemento l'aggettivo bello/bèlo (come Borgobello, Montebello e simili). Oppure trovare origine nelle innumerevoli e ben attestate immigrazioni di elementi dalmati e balcanici verso le coste italiane, dalla Puglia a Venezia.

17 Soprannome di un ramo dei gemonesi Goi, vecchio cognome locale, secondo lo scrivente anch'esso di origine slava.

18 Gli abitanti sono conosciuti con questo etnico per la vicinanza del Bíli Putók/Rio Bianco (Dapit 1998: 141).

19 Cfr. anche l'antropotoponimo croato Belanovo Selo (ad ovest di Koprivnica, Hr).

20 È noto come il fondo slavo medievale sia ben presente nella toponomastica e nell'antroponimia romene, anche in cognomi con formante -an, come Bejan: es. Birsan, Bolozan, Pervan, Prodan ecc.

21 Nella periodizzazione slavistica il tardo periodo della cosiddetta fase slava-alpina (precedente a quella slovena).

22 Si trattava di una «comunità impegnata ad accudire un "santuario», piuttosto che... un cenobio di regola benedettina o... una abbazia vera e propria»; a S. Canziano era conservato il Vangelo di S. Marco, visitato da pellegrini provenienti da varie regioni dell'impero carolingio ma anche da lontane regioni slave come la Bulgaria (Toplikar/Tavano 2005).

23 Onomastica: XIV sec. Rodis; Volthas; XV sec. Antonius q. Juvan de Crapina, Anzeli Breganti, Michael Babaz, Blasii Gorsii, Gregorius Cocevar, Johannis Collarich, Cotian, Gregorius Drobench, 
A sud di Pieris e Begliano si allargava un territorio compreso fra un ramo medievale dell'Isonzo ${ }^{24}$ il fiume Sdobba (da un composto slov. $i z-+d o b$ 'quercia') ed il mare; era infatti chiamato dai friulani Isola. ${ }^{25}$ Durante il medioevo fu sempre diviso fra i grandi boschi a nord e la palude a sud e fu dalla metà del sec. XII alla fine del sec. XV di proprietà dell'abbazia di Moggio. Almeno da quando, fra gli anni 1136 e 1188, fu donata ai benedettini dai signori di Chiarisacco Werner e la moglie Berta, probabile famiglia di ascendenza arimannica longobarda (Wiesflecker I, 287).

L'Isola non aveva abitati stabili ma era frequentata da boscaioli e pescatori che pagavano un censo agli agenti dell'abate. Finora non erano emersi documenti che riportassero nomi del periodo medievale, antecedente al grande esproprio veneziano della fine del sec. XV. A cominciare dal sec. XVI vi fu un progressivo popolamento con contadini provenienti da varie parti, che rinnovarono quasi del tutto la toponimia.

Da ricerche svolte negli ultimi quattro anni risultano confermati il nome principale sloveno per Isola, Otoch ${ }^{26}$ e tre toponimi della vasta selva: *Martiniza, ${ }^{*}$ Stradoniza, e Studens. Le ricostruzioni dipendono dall'esasperante imprecisione della nomenclatura riportata in molti documenti veneziani. ${ }^{27} \mathrm{E}$ sono emersi altri toponimi di questa grande selva isontina chiamata dagli sloveni del Monfalconese *Otok e dai friulani di Fiumicello Isula. Acque come il gurgus nominatus Martin vir ('gorgo di acqua sorgiva' detto «di Martin»), Ara de Martin vir seu Martignana ('canale della sorgente di Martin detto anche Martignana'28), Ara magna Studenshic ('canale grande degli Studenzi' ${ }^{29}$ ), Ara que intrat ... appellatur Ulzach ('canale confluente... detto Ulzach'), Ara de Otoch

Hierman tabernarius, Iorium supanum Thomasii, Janes, Jurius, Jury de grozdio, Justo dicto Cucus, Lucman rodarius, Male, Michaelis Besech, Puntel Gorse, Stefanum dictum Cos, Simez Sicollino, Stanchi, Stefanus boscator sclavonus, Voricum quondam Simiç, Zorzi Bosegniach; XVI sec. Blanchichio, de Cobalico, Cocus, Jurinet, Primus Norich, Simiz, Stanchich, Voucichio, Zanutichz. Toponimi: Babbiza, Bosovizza, Bravizze, Brechi, Clapece, Dobia, Gorischizza, Grode, Gubilinizza, Gurizzizza, Latoch, Melacha, Novacuz, Pan, Presecha, Suemarisca, Vela, Verbilico, Vinogradi, Vorich, Voucicoti (P 2010).

24 Ora in gran parte continuato dal canale Isonzato (forma peggiorativa romanza di Isonzo).

25 Dopo l'esproprio veneziano vi fu il passaggio successivo nelle mani di signori veneti, Malipiero, Morosini (da cui il nome attuale di Isola Morosini) e triestini (Brunner). Altri nomi della zona furono Pietrarossa (dal nome della pietra confinaria veneziana dipinta di 'rosso') e Di là dell'Ara alludendo al fatto che era situata «di là del fiume Sdobba» (detto nel dialetto veneto moderno del Monfalconese anche Àra 'canale').

26 Luogotenente alla Patria del Friuli, b. 59, Processi 1477-78, fl. ‘1478', cc. 230-31 (ASVe).

27 Es. Martincha, Martinicha, Mortanizza, Martonizza, Martinuza; Stradonicha; Stuidan, Studens (b. 273, Ducali 1483-1492, c. 192r \& v.; b. 88, Processi ed Investiture, 1489-1490 (ASVe); Congregazioni Religiose Soppresse, Moggio, ASUd).

28 Martignana potrebbe essere la denominazione friulana della territorio detto altrove Martiniza, usata forse dalla gente di lionguaggio romanzo, che seppure minoritaria è attestata in zona.

29 Ara è un termine romanzo indicativo di un 'canale di palude'. Studensi è il nome di località campestri diverse poste a sud di S. Canziano. 
('canale di Otoch'/Isola). ${ }^{30}$ Da notare i toponimi sloveni Martin vir, *Studencih ('alle sorgenti'), *Olšah ('agli ontani') e Otoch.

Le realtà fisiche e fitonimiche sono oggi inidentificabili perché tutta l'area bassoisontina è stata sconvolta dal raddrizzamento dell' asta fluviale dell'Isonzo (sec. XIX) ${ }^{31}$ e dalle grandi bonifiche avvenute negli ultimi due secoli.

Si è già detto che l'importanza di Pieris era determinata dalla presenza del traghetto sul fiume Isonzo, denominato in tutti i documenti medievali Zopum. ${ }^{32}$ Questa è la forma latinizzata del termine fr. çop (e zàup, zop, zòip, con forme diminutive zòpul e anche zopolèt nel goriziano del XVIII secolo ${ }^{33}$ ), indicativo di un natante scavato in un tronco d'albero. Il termine ed il suo uso sono ben testimoniati nella documentazione sinora emersa: 1356 Stoyna de Begliano ultra Ysoncium... pro navicula seu zopo fluminis Ysoncii (STC 15). L'etimo di fr. zop era alquanto controverso ma la discussione si è rinfocolata dopo la pubblicazione di un volume del Volpi Lisjak sulle čupe ${ }^{34}$ in uso fra i pescatori sloveni della costiera triestina. Secondo quest'autore la voce slovena (limitata al Triestino) non avrebbe alcun rapporto con la friulana bensì con una nota nella Russia settentrionale, sul lago Ladoga. Il russo čupa, čupka, čupocka (con i derivati čupas e čupus) col significato base di 'legno cavo o vasca-contenitore, serbatoio' mostra effettivamente una somiglianza formale con l'isolato termine sloveno, ma va considerato che nella regione del Ladoga il sostrato finnico è molto forte e che nel lappone suves, so'ppas, subpas sta per 'chiglia'. Per cui il termine russo potrebbe continuare benissimo una voce del sostrato finnico. ${ }^{35}$

In tutta la vastissima area delle lingue slave il semantema 'piroga, tronco scavato' si esprime con altre voci, affini alle slovene čôln e drevak/deblak (Snoj 2003: 89-90, $97,123) .{ }^{36}$

Nelle regioni adriatiche settentrionali emergono due ipotetici fuochi d'irradiazione, ove un termine base çop/zop-/zaup- vanta attestazioni medievali: uno in Dalmazia ('barca, ligno vel zaupo' ad Arbe/Rab nel XVI secolo; Sella 1944: 633). Ed uno in Friuli sui passi dell'Isonzo (1371 de transitu seu Zopo quod est super aqua Isontii

30 Luogotenente alla Patria del Friuli, b. 59. Processi 1477-1478, fl '1478', c. 230r >c. 231v. (ASVe). Il termine àra ('canale' in zone paludose), diffuso nelle Basse del Veneto e del Friuli, deriva dal latino area, che ha avuto un'evoluzione complessa, qui non sintetizzabile.

31 Portato a termine dal governo austriaco a fine ' 800 .

32 Erano quattro i passi con traghetto nel basso Isonzo: Fogliano, Cassegliano, Pieris e Isola (quest'ultimo di importanza solo locale).

33 S'ingiavin fur dal zoc ju zopolèz 'si ricavano dal ceppo d'albero le piroghe', nell'Eneide di Virgilio nella traduzione in friulano goriziano settecentesco del Bosizio (Bosizio: 17, riportato in NP: 1320).

34 In Pleteršnik (1894: 109), čopa.

35 In quell'area erano attestati i gruppi etnici Vepsi e Ciudi, di lingua finnica.

36 La prima voce, panslava, deriva da una nota base indoeuropea ed indicava in origine un 'tronco segato, abbattuto; le ultime due voci insistono sul semantema 'tronco d'albero', esattamente come il fr. çop. 
super locum qui dicitur de Foglano). ${ }^{37}$ Altri toponimi friulani e alto-veneti con apparente affinità sono molto meno sicuri poiché vi concorrono basi simili di valenza semantica differente: Zoppola-Pn (di antica attestazione ${ }^{38}$ ), Zupignes di Tramonti di Sopra-Pn, Zupigne di Timau-Ud, Zoppè (Bl, Tv) ecc. ${ }^{39}$

Da notare che in un registro medievale cividalese (Libro degli anniversari del convento di S. Domenico) troviamo un Nicolaus Çuparius per l'anno 1337 (Scalon II, 19: 687): forse era il nome «di mestiere» di persona o famiglia impegnati nella costruzione di queste imbarcazioni arcaiche.

Come abbiamo visto i due centri dove si rintracciano le attestazioni più antiche e semanticamente sicure ('piroga') ${ }^{40}$ sono situati nel basso Friuli orientale e in un'isola della Dalmazia centrale, dove probabilmente si parlava ancora l'antica lingua dalmatica. Mentre le attestazioni dello sloveno triestino čupa non vanno oltre il sec. XIX, così che si è portati a pensare ad un prestito dall'antico dialetto ladino di Trieste. Nel friulano con la scomparsa degli ultimi esemplari di piroghe durante il sec. XVIII anche il termine è stato dimenticato. ${ }^{41}$ Ma il significato base di 'tronco, ceppo' lo ritroviamo ancora in alcune voci agrarie e rustiche del Friuli e del Veneto settentrionale ed orientale: fr. ciòp 'ceppo' (NP: 1471), fr. zòbare 'ceppaia... grosso pezzo di legno rinsecchito', fr. zòpare 'pezzo grosso tagliato dal ciocco' (NP: 1676, 1677) ${ }^{42}$ fr. zupièl 'sorta di zoccolo' (NP: 1327), pavano antico zuoppo 'ceppo', zopello 'zoccolo' (Prati EV: 210; Paccagnella: 894), veneto moderno zopèla/sopèla 'zoccoletto, ciabatta rustica', ${ }^{43}$

371356 navicula seu zopo...; 1371 de transitu seu Zopo...; 1393 transitum sive zopum super aqua Usoncij; XV sec. Zopi (STC 15; di Prampero: 60; Puntin 2010: 199; NP: 1320).

381103 Zaupola, 1186 de Zaupolis (Petris 2014: 87). Mancando importanti corsi d'acqua in zona, secondo il Petris il top. Zoppola (fr. Sòpula) rimanderebbe al fr. ciàup 'truogolo' (in origine ligneo, NP: 142).

39 Zompicchia di Codroipo-Ud (fr. Çupicje) e Zompitta di Reana-Ud (Çumpite) potrebbero continuare un antico personale sloveno Zopic (*Copik) attestato in carte medievali friulane. Altri (soprattutto i microtoponimi agrari) si spiegheranno con friulano e panveneto zòpel zòpa/sòpa/thòpa 'zolla'.

40 Nel Veneto l'uso delle piroghe è naturalmemte attestato in fonti medievali, ma la Cronaca Altinate del sec. XI ha il termine monosolum (< lat. monoxylus) o quello generico di 'tronco cavo' (in lignis caveis, in caveis lignorum; Cronaca I: 194).

41 In documenti e scritti dei secoli XVII e XVIII si registrano queste tre forme: zoipo (*zòipo), zopul

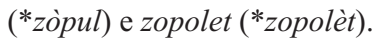

42 Probabile evoluzione di un diminutivo *zòpula (<*zòpura), come fr. àlbare ('pioppo bianco') da lat. albula.

43 In origine questi termini potevano aver indicato in alcune zone gli zoccoli arcaici consistenti in un legno incavato (come le dàlminis carniche). Ma col passare dei secoli e la scomparsa di questo vecchio tipo di calzatura, ha finito per denominare in molte aree del Veneto anche zoccoli aperti e piani e addirittura ciabatte rustiche e simili. Dalla calzatura proviene forse anche il cognome veneto e friulano Zuppel. 


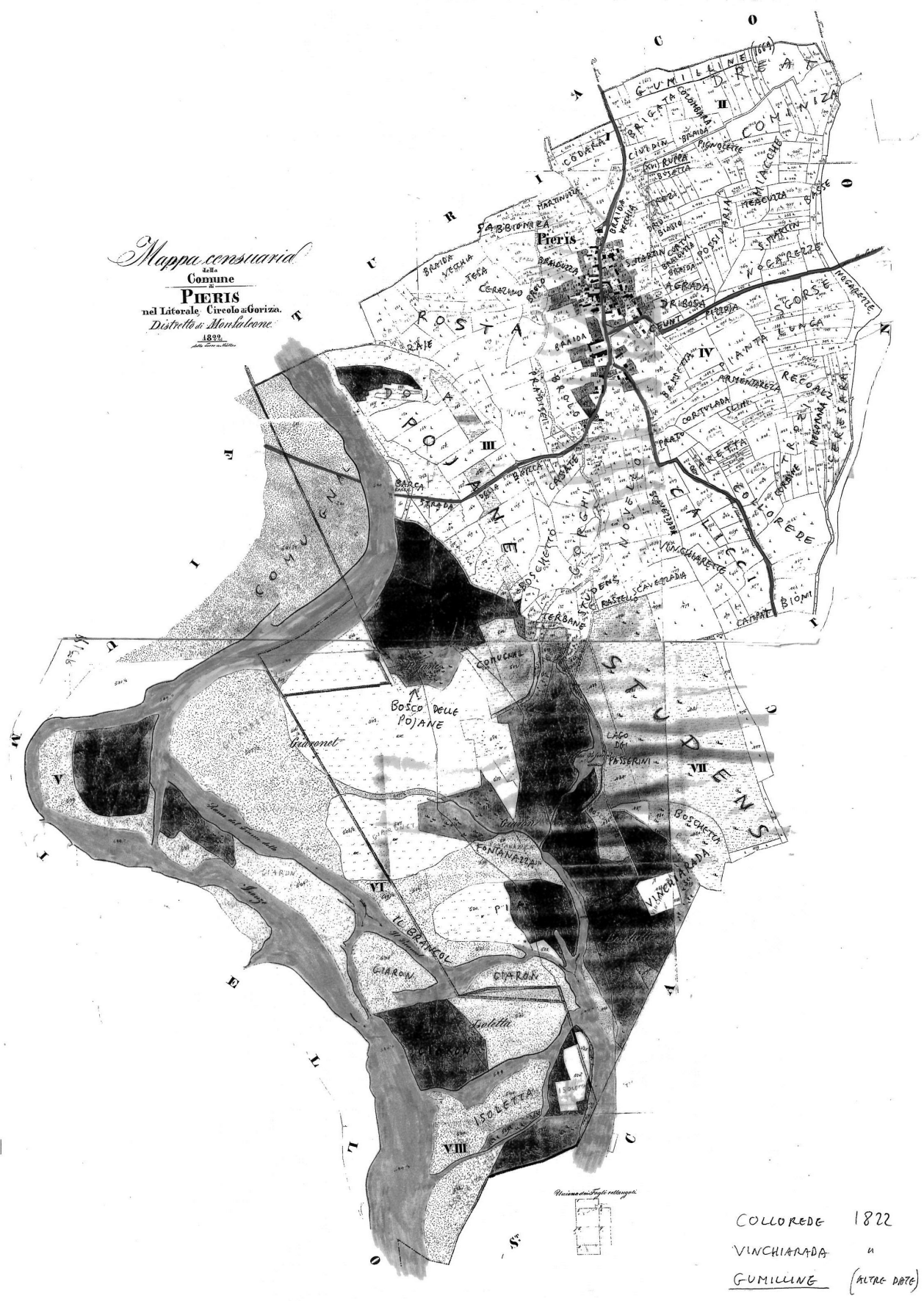


Tornando alla base che emerge chiaramente dalle attestazioni più antiche, *zaup-/*zop-e mancando appigli nella lingua latina, ${ }^{44}$ sembra plausibile l'ipotesi di una voce prelatina ${ }^{45}$ Diffusa specialmente nella Venetia orientale e nella vicina Dalmatia, questa voce sarebbe passata al latino provinciale e poi adattata variamente nei continuatori romanzi ed in quelli sloveni adriatici.

\section{Fonti primarie ${ }^{46}$}

Congregazioni Religiose Soppresse, (Abbazia di) Moggio (ASUd = Archivio di Stato di Udine).

Cronaca Altinate (in latino, di Anonimo di Altinum)

Le note di Guglielmo da Cividale (1314-1323); Gianni Luca (a cura di) (2001)

Luogotenente alla Patria del Friuli - b. 32, Processi 1462/1464, c. 100r; b. 59, Processi 1477-78, fl. '1478', cc. 230-31; b. 273, Ducali 1483-1492, c. 192r \& v.; b. 88, Processi ed Investiture, 1489-1490 (ASVe = Archivio di Stato di Venezia).

$\mathrm{SAC}=$ Schedario Antroponimico di G.B. Corgnali (in Biblioteca Civica di Udine «Joppi») STC $=$ Schedario Toponomastico di G.B. Corgnali (in Biblioteca Civica di Udine «Joppi»)

\section{Fonti secondarie}

$\mathrm{NP}=$ Il Nuovo Pirona Vocabolario friulano, 1992

P 2005 = Puntin M., Slovenci na tržiškem Teritoriju-Obdobje med 7. in 17. Stoletjem, 2005.

P 2010 = Puntin M., Dei nomi dei luoghi. Toponomastica storica ..., 2010

\section{Bibliografia}

BILLY Pierre-Henri (2011) Dictionnaire des noms de lieux de la France. Saint Etienne: Editions Errance.

DAPIT, Roberto (1998) Aspetti di cultura resiana nei nomi di luogo, 2. (Area di Oseacco e Uccea.) Padova: CLEUP.

DI PRAMPERO Antonino (1882) «Glossario Geografico Friulano, dal VI al XIII secolo.» Atti del Regio Istituto Veneto di Scienze Lettere e Arti, serie V, voll. VII-VIII, 1-236

GIANNI, Luca (a cura di) (2001) Le note di Guglielmo da Cividale (1314-1323). Udine: Istituto Pio. Paschini, Udine.

44 Qui si escludono etimologie fondate su lat. cüpa o lat. volgare cōpa ('botte') o su lat. cymba ('barca'), come proposto in passato da certi autori. I passaggi morfologici non sono assolutamente plausibili.

45 Forse da una radice *tsaup- ('tronco, ceppo'). Per una ampia discussione: Pellegrini (1992).

46 L'autore dell' articolo è debitore per alcune informazioni e per i risultati di alcune recenti ricerche d'archivio (in Archivio di Stato di Venezia) a Carlo Mucci e Diego Mauchigna. 
KRANZMAYER, Eberhard (1956-58) Ortsnamenbuch von Kärnten. I-II. Klagenfurt: Geschichtsvereines für Kärnten.

KRONSTEINER, Otto (1981) Die Alpenslawischen Personennamen. Wien: Österreichische Gesellschaft für Namenforschung. (Sonderreihe, 2.)

MERKÙ, Pavle (1996) «Predkrščanska slovenska osebna imena v Trstu (1307-1406).» In: J. Kos et al. (a cura di), Brižinski spomeniki. Ljubljana: SAZU, ZRC SAZU, 451-455.

MERKÙ, Pavle (2001) «La datazione dei toponimi slavi in Friuli: problematiche e ipotesi.» In: Atti del $14^{\circ}$ Congresso internazionale di Studi sull'alto medioevo «Paolo Diacono e il Friuli altomedioevale (secc.VI-X). (Cividale del Friuli-Bottenicco, 24-29 settembre 1999). Spoleto: Centro italiano di studi sull'Alto Medioevo, 697-712.

PACCAGNELLA, Ivano (2012) Vocabolario del pavano (XIV-XVII secolo). Padova: Esedra.

PELLEGRINI, Giovan Battista (1992) Studi di etimologia, onomasiologia e di lingue in contatto. Alessandria: Edizioni dell'Orso.

PETRIS, Nerio (2014) Stradis. S. Vito al Tagliamento. (Quaderni Zoppolani, 12.)

PIRONA, Giulio Andrea/Ercole CARLETTI/Giovanni Battista CORGNALI (1992) Il Nuovo Pirona Vocabolario friulano. Seconda edizione con Aggiunte e correzioni riordinate da Giovanni Frau. Udine: Società Filologica Friulana.

PLETERŠNIK, Maks (a cura di) (1894-95) Slovensko-nemški slovar. I-II. Ljubljana: Knezoškofijstvo.

PRATI, Angelico (1968) EV - Etimologie venete. Opera postuma a cura di Gianfranco Folena e Giovan Battista Pellegrini. Venezia/Roma: Istituto per la collaborazione culturale.

PUNTIN, Maurizio (2005) «Slovenci na tržiškem Teritoriju. Obdobje med 7. in 17. stoletjem.» In: V. Klemše (ur.), Slovenci v Laškem Cenni storici sulla comunità slovena nel Monfalconese. Tržič/Gorica: SKŠRD, 15-60.

PUNTIN, Maurizio (2009) «La prima colonizzazione slava del Friuli nella toponomastica.» Dom, nn. 17, 18, 19, 20, 21, p. 3.

PUNTIN, Maurizio (2010) Dei nomi dei luoghi. Toponomastica storica del territorio di Monfalcone e del comune di Sagrado. Gradisca: Centro Leopoldo Gasparini.

SCALON Cesare (a cura di) (2008) I Libri degli Anniversari di Cividale del Friuli I-II. Roma/Tavagnacco (Ud): Istituto Storico Italiano per il Medio Evo/Istituto Pio Paschini. SELLA Pietro (1944) Glossario latino italiano. (Stato della Chiesa-Veneto- Abruzzi.) Città del Vaticano, Roma: Biblioteca Apostolica Vaticana.

SNOJ, Marko (2003) Slovenski etimološki slovar. Ljubljana: Modrijan.

TOPLIKAR Giovanni/Sergio TAVANO (a cura di) (2005) I Santi Canziani nel XVII Centenario del loro martirio - Sveti Kancijani ob 1700-letnici mučeništva. Ronchi dei Legionari: Consorzio Culturale del Monfalconese.

TORKAR, Silvo (2007) «Toponim Preserje in slovansko-romansko prepletanje pripon -jane in -anum.» In: S. Torkar et al. (a cura di), Merkujev zbornik. Založba ZRC, 481-492. 
VOLPI LISJAK, Bruno (2004) Prvo slovensko plovilo in drevaki. Prispevek $k$ etnologiji in vprašanju etnogeneze Slovencev. Mladika: Trst.

WIESFLECKER, Hermann Paul (1949/1952) Die Regesten der Grafen von GörzTirol, Pfalzgrafen in Kärnten. I -II. Innsbruck: Wagner.

\section{Riassunto \\ PIERIS E BEGLIANO: VILLAGGI MEDIEVALI DEL BASSO \\ ISONZO DALL'INCERTA IDENTITÀ}

L'autore ha studiato a fondo, a cominciare dagli anni '90, la toponomastica e l'antica antroponimia del Territorio di Monfalcone. Dopo la pubblicazione della II edizione dell'opera (P 2010) le ricerche sono continuate e hanno sempre confermato quei primi risultati. In pratica questo angolo sud-orientale del Friuli rientrò almeno fino a tutto il sec. XV nella Slavia submersa, con una maggioranza di abitanti slavofoni ed una minoranza parlante un dialetto friulano che si situava morfologicamente fra quello centrale e le antiche e scomparse parlate friulaneggianti di Trieste e Muggia. Nell'articolo si ripresentano brevemente molti nomi di persone e di luoghi di Pieris e Begliano (oggi Comune di S. Canzian d'Isonzo), con alcuni nuovi dati emersi ultimamente: per esempio sull'attuale località Isola Morosini (nel sec. XV Otoch) che apparteneva in età medievale all'Abbazia benedettina di Moggio. Viene rivista anche l'etimologia del nome della località di Begliano, lasciata in sospeso nei lavori precedenti fra l'opzione predialistica romana $\left({ }^{*}\right.$ Bellius $)$ e quella paleoslava $\left({ }^{*}\right.$ Beljan o *Beljani $)$; assegnando questo toponimo allo strato linguistico slavo.

Bisogna distinguere però questo strato sloveno medievale da uno successivo, rappresentato essenzialmente da nomi e soprannomi di persone immigrate nel Monfalconese fra la fine del sec. XV e il sec. XVII. Gente di origine balcanica (sclabonus sive bisiacus) che fuggiva dalle invasioni turche ed entrava in un angolo di Friuli soggetto a Venezia.

I tre villaggi erano vicini all'antico traghetto (zopum) di Pieris sul fiume Isonzo e la parte finale dell'articolo è dedicata alla discussione sull'etimo del termine friulano çòp/ zòpul 'piroga' (slov. dial. čupa), su cui era intervenuto uno scrittore di Trieste proponendo un'origine slovena. L'autore dell'articolo mostra invece come non si possa facilmente disgiungere il termine çop/zop da tutta una trafila di voci, ben attestate nell'area romanza del nord-est italiano (compresa la lingua dalmatica oggi estinta). Voci che sembrano tutte gravitare attorno al semantema "ceppo, legno scavato" e che potrebbero in ultima analisi essere di lontana origine prelatina.

Parole chiave: bel ('bianco'), bezják (abitante del Monfalconese), isola, Isonzo (fiume), zop ('piroga') 


\section{Abstract \\ PIERIS AND BEGLIANO: \\ MEDIEVAL SETTLEMENTS OF THE LOWER SOČA OF UNCERTAIN IDENTITY}

The author has studied in depth, starting from the 90s, the place names and the ancient anthroponymy of the Monfalcone area (the old Ultraisoncium 'beyond the Isonzo river'). After the publication of the second edition of his monograph, the research continued and confirmed the initial results. In practice this South-eastern corner of Friuli came back at least until the end of teh $15^{\text {th }}$ century to Slavia Submersa, with a majority of the inhabitants Slovene speaking and a minority speaking a Romansh language that ranged morphologically between the central Friulian and the old Romansh dialect of Trieste. The article presents some new data on the three neighbouring countries of the old ferry (zopum) Pieris on the Isontium river; and a discussion of the etymologies of the toponym Begliano (assigned permanently to the Slavic layer) and the Friulian term çop/zòpul 'pirogue' (Slovene dialect čupa), giving more emphasis here for Romance origin.

Keywords: white, bezják ('inhabitant of district of Monfalcone'), island, Isonzo (river), zop (Friulian word: 'pirogue')

\section{Povzetek \\ PIERIS IN BEGLIANO: SREDNJEVEŠKI NASELJI OB SPODNJI SOČI Z NEJASNO IDENTITETO}

Od 90. let prejšnjega stoletja dalje se avtor poglobljeno ukvarja s krajevnimi imeni in s starimi antroponimi območja okoli Tržiča (Monfalcone), znanega tudi kot Ultraisoncium 'območje onkraj reke Soče'. Po objavi druge izdaje monografske predstavitve tematike so se raziskave nadaljevale in večkrat potrdile začetne rezultate. Ta jugovzhodni del Furlanije je bil dejansko vsaj do konca 15. stoletja ponovno vključen v ozemlje, znano kot Slavia Submersa (nekdanje slovansko ozemlje), kjer je večina prebivalcev govorila slovensko, manjšina pa alpsko romansko narečje, ki je bilo v oblikoslovnem pogledu med osrednjo furlanščino in alpskim romanskim narečjem v Trstu in Miljah. V članku avtor na kratko ponovno razpravlja o številnih osebnih in krajevnih imenih, ki jih nademo v Pierisu in Beglianu (danes v občini Škocjan ob Soči) in navaja nekaj nedavno odkritih podatkov, npr. v zvezi s krajem Isola Morosini (v 15. stoletju Otoch), ki je v srednjem veku spadal pod benediktinsko opatijo v Možacu.

Avtor prikazuje nekaj novih podatkov o treh sosednjih vaseh ob rečnem prehodu (zopum) v Pierisu na reki Soči in razpravlja o etimologiji toponima Begliano, ki mu pripisuje slovanski izvor, in furlanskega izraza çop/zòpul 'čupa', ki pa naj bi izhajal iz romanske osnove. Ponovno pretresa tudi etimologijo imena kraja Begliano, ki je v 
doslejšnjih raziskavah ostala nedorečena, kot mogoči pa sta se ponujali dve razlagi: po prvi naj bi ime izhajalo iz romanskega osebnega imena (*Bellius), po drugi pa iz staroslovanskega $(*$ Beljan ali *Beljani). Po avtorjevem mnenju je izvor imena treba iskati v slovanski jezikovni plasti. To slovensko srednjeveško plast pa je treba razločevati od mlajše plasti, ki jo predstavljajo predvsem imena in vzdevki oseb, priseljenih na tržiško območje med koncem 15. stoletja in 17. stoletjem. Gre za ljudi balkanskega izvora (sclabonus sive bisiacus), ki so bežali pred Turki in se naselili v koščku Furlanije, ki je bil pod beneško oblastjo.

Omenjene tri vasi so bile blizu rečnega prehoda (zopum) v Pierisu na reki Soči in zadnji del prispevka se ukvarja z izvorom furlanskega izraza çòp/zòpul, glede katerega je neki tržaški avtor predlagal, da je slovenskega izvora. Iz pričujočega članka pa je razvidno, da ni mogoče zlahka ločevati med izrazom çop/zop in vrsto drugih besed, ki so dobro izpričane na romanskem območju italijanskega severovzhoda pa tudi v danes izumrlem dalmatskem jeziku. Vse te besede so povezane s pomensko osnovo 'drevak, izdolben kos lesa' in bi lahko bile daljnega predlatinskega izvora.

Ključne besede: bel, Bezják (prebivalec Tržiča in okolice), otok, Soča (reka), zop (čupa) 